\title{
Role of electrostatics in artificial pollination and future agriculture
}

\author{
Dipak S. Khatawkar ${ }^{1, *}$, Shaji P. James ${ }^{2}$ and D. Dhalin ${ }^{3}$ \\ ${ }^{1}$ Department of Farm Machinery and Power Engineering, Kerala Agricultural University, Thrissur 679 573, India \\ ${ }^{2}$ Agricultural Research Station, Mannuthy 680 651, India \\ ${ }^{3}$ Department of Agricultural Engineering, College of Agriculture, Vellayani 695 522, India
}

Pollination is the process of pollen detachment from the male part, i.e. anther of a flower, travel through a medium and deposition onto the female part, i.e. pistil of flower, thereby enabling fertilization and reproduction. Majority $(87.5 \%)$ of flowering plants rely on animal pollination, on insects in particular, with the remnants of species being wind-pollinated and with autonomous seed-producing capabilities. The reproductive phase of a plant has direct impact on crop yield and quality of produce in terms of biomorphological characters which affect its market value. On a global account, $80 \%$ of all plant species depend on pollination for reproduction, which influences $35 \%$ of crop production and worth US\$ 117 billion per year. However, drastic decline in native pollinators has raised major concerns for the assurance of pollination services. Studies have revealed that the absence of pollinators may reduce the global agricultural crop production by 3-8\%. Also, keeping in mind the world scenario of crop production and population expansion in relation to land under cultivation which remains the same; rather it shows a decreasing trend. Crop productivity has to be optimized from all possible points of view to meet the future food demand. Here the concept of precision agriculture arises, i.e. optimum utilization of available resources to achieve maximum possible yield from the same land area and desirable quality, without jeopardizing the environment. If the pollination process is disturbed due to climatic factors or insufficient pollinators during the period of bloom, it may directly result in lower yield, undesirable biomorphological changes and quality loss, leading to mass economic drop. To overcome this situation, artificial pollination techniques have been developed.

Keywords: Artificial pollination, crop production, electrostatic charging, food security, honey bee.

\section{Natural pollination}

POLLINATION is an important phenomenon in the context of most of the plant species enabling them to reproduce to continue their biological life-cycle. Almost two-third por-

*For correspondence. (e-mail: dipakkhatawkar@gmail.com) tion of angiosperms are dependent on vectors as animals and insects ${ }^{1-3}$, with the remainder of species being either wind-pollinated or completely reliant on autogamy ${ }^{4-7}$. From the economic point of view pollination is one of the major influencing factors apart from the other cultivation practices like soil manipulation, nutrients, disease and pest control, irrigation, etc. Since pollination is the key factor in reproduction system of the plant, it has direct impact on crop yield, quality of produce in terms of biomorphological characters and sensory evaluation which in turn influences market value of the produce ${ }^{8-11}$.

\section{Artificial pollination}

Pollinators influence a huge monetary share $(75 \%$, i.e. US\$ 117 billion approx.) annually in the worldwide agricultural produce sector. Even a small hindrance to the pollinators' population, generates the well known 'butterfly effect', which causes severe depletion (3-8\%) in the agricultural output in subsequent stages ${ }^{12}$. Since more than a decade, this has been a major concern for the scientific community across the planet to find sustainable solutions for assuring a healthy population of natural pollinators. The food security of ever expanding world population and future sustenance of mankind, strict actions are necessary to protect natural pollinators as well as improve techniques of artificial pollination to meet future demands. In the era of Agriculture 4.0, we must be more focused on sustainable and greener solutions ensuring environmental and ecological safety, rather than production oriented approach alone. Pollination is a delicate process in nature and is vulnerable to many external factors such as, man-made high-frequency telecommunication networks as well as harsh climatic conditions like wildfires, cyclones, etc. To sustain in this situation artificial pollination techniques have been developed and being used from past few decades, are reviewed comprehensively in this article.

\section{Hand pollination method}

Artificial pollination, including manual detachment of pollen grains from anther, collection and storage, and at 
the time of female blooming depositing them onto the receptive stigma by hand or using suitable medium for dispersal of the pollen grains is hand pollination. The degree of pollination depends upon the viability of pollen and their deposition level on the targeted plant organ. A trained and skilled person alone can carry out this work, as it needs much care and precision to pollinate successfully. For example, China is the global leader in apple production. However, it has seen a sharp decline in native pollinator abundances which has become an important field of research in the past decade. The Italian bee (Apis mellifera ligustica), which accounts for $61.5-99.4 \%$ of overall pollinators, is observed to be reduced in population and activity as well due to climate change and pesticide use ${ }^{13}$. Farmers are forced to pollinate the apple flowers manually to ensure fruit yield. It is true that the hand pollination method can never serve as the mainstream pollination service for large-scale production, owing to the fact that all flowers cannot be pollinated manually to obtain the desired yield in order to meet the huge global demands. Also, it is a delicate and monotonous method, which is practically cumbersome and uneconomical on account of labour requirement.

In order to cover large orchard areas, it is necessary to have a medium to carry pollens grains over large areas and to reach crowns of the tall trees, such as date palm. For this, prevailing wind or artificial air blast has been practised over a long period of time.

\section{Mechanical pollination}

For pollinating tall orchard trees mechanical devices have been developed which enable pollination of the plants that are more than $2.5 \mathrm{~m}$ high and generally considered out of vertical reach of normal human beings. Artificial pollination done with the help of such mechanical devices is known as mechanical pollination. These devices are generally made of long telescopic light metal or plastic tubing to reach the target as well as to reduce handling load on the operator. This type of device has been developed for artificial pollination of date-palms by Vaknin et $a l .{ }^{14}$ at Tel-Aviv University, Israel (Figure 1). This mechanical equipment has a telescopic lance consisting of an aluminium tube with a plastic delivery tube tied to it, where dried pollen bottle is placed at the bottom end with appropriate pumping-type hand-blower device. Under working condition, the operator has to adjust the stretch of the telescopic lance according to the height of the palm tree at which the target is situated. Once the lance has been set near to the target, dried pollen grains are pumped from the bottle by creating air pressure which makes the pollen to disperse from the bottle to the target through the plastic tubing. The assembly has one more metallic attachment to the top of the lance having a sharp hook shape used to slit open the female spathe during early stage of bloom. It helps to create more exposed area of female spathe to deposit the pollens.

However, the developed mechanical tool incurs more drudgery to the operator while adjusting and holding the lance vertically and seems to be tedious while working with date-palm orchards spread over tens of hectares. Also, it becomes difficult to handle the lance, in a stable position when the height is beyond 3-4 m, due to inertia forces and prevailing winds. Therefore, trees of height beyond $5 \mathrm{~m}$ are extremely difficult to work with, and this shows the need for more sophisticated and effective method to deliver pollen to the target height. The existing methods are difficult, require large quantities of pollen for achieving sufficient level of deposition and have been proved to be time-consuming methods. Also, the wastage of pollens becomes uneconomical for bigger orchards. To minimize the pollen requirement and optimum deposition over the targets, an alternative technique has to be adopted.

\section{Electrostatics in artificial pollination}

Electrostatically charged particles have a tendency to get attracted towards the oppositely charged object. The electrophoretic effect has already been applied fruitfully in automotive surface-coating processes, agro-chemical sprayers and dust suppression. During manual pollination there is chance of physical damage to the pollen grains while detaching them from the anther by hand or any mechanical tool. This early-stage damage can result into failure or inferior germination in future and degrade the quality of the products.

Pollination is a factor of prime importance in achieving satisfactory crop yield. Lower yield in the field as well as horticultural crops has been frequently reported due to insufficient or inferior pollination ${ }^{15,16}$. Pollination is a key

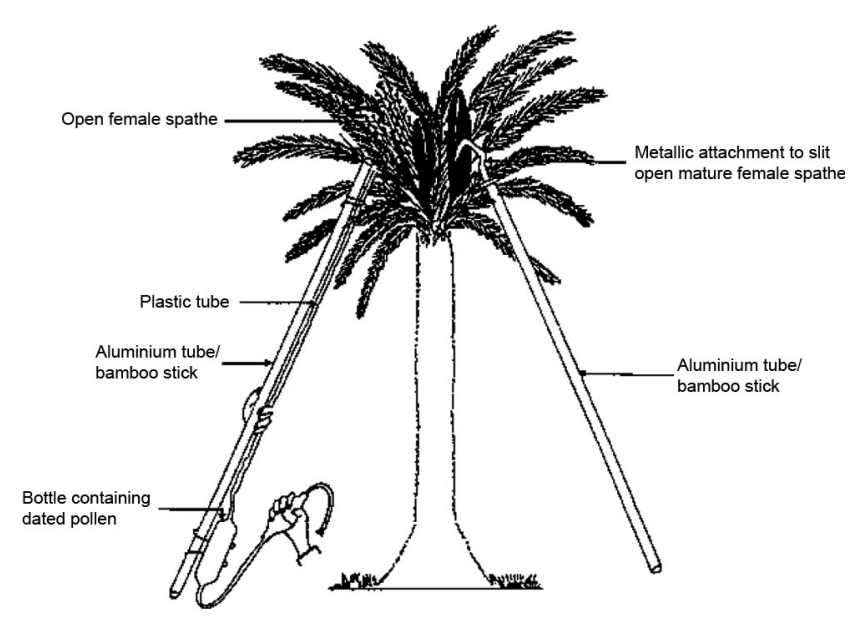

Figure 1. Mechanical pollination equipment for date palm (source: ref. 14). 
part of the plant reproductive system. From the viewpoint of precision agriculture and competitive market scenario across the globe, pollination has a significant role in production of quality fruits, vegetables and seeds having desired characteristics. Along with the general cultivation practices such as the root-zone nourishment, soil moisture, pest and disease control, and other intercultural practices, crop productivity radically depends on the level of pollination. Inferior pollination results into unsatisfactory fruit form, size and morphological homogeneity, which in turn decreases its value in both in-land and overseas markets ${ }^{17-19}$.

Artificial pollination requires skill and specialized equipment to collect pollen grains from the anther, drying and depositing them onto the receptive stigma without mechanical damage ${ }^{20}$. Nectarless-flower plants are more susceptible to high rate of pollen loss due to insufficient pollinators and pollen-stealing visitor insects. Such species need to be protected from pollen loss and pollinated by artificial means ${ }^{21}$.

The role of electrostatics in natural honey-bee pollination has been explored and confirmed by researchers as a significant factor in pollen detachment in the last two decades. Studies exposed that post-flight charge accumulation on the bee was $23.1 \mathrm{pC}$. The observed forces required for detachment of pollen were in the range $4 \times 10^{-10}-39 \times 10^{-10} \mathrm{~N}$ for selected horticultural species. Scientific modelling and analysis showed that there were conceivable probabilities where the accumulated charge on a honey bee was capable enough to detach pollen without physical contact ${ }^{22}$. The average vertical electric field could be $100-150 \mathrm{~V} \mathrm{~m}^{-1}$, while ground surfacecarried negative charge and air possess equal and opposite positive charge. As the plants are grounded to earth, they carry negative charge, but this charge is found to be uneven on the plant body, especially concentrated near the tips and spiked portions ${ }^{23,24}$

Studies on application of electrostatic pollination technique have been carried out for wind-pollinated crops like date palm, which showed significant rise in fruit set due to increased deposition of charged pollen, while reporting less amount of pollen required to pollinate sufficiently $^{25,26}$.

\section{Methodology}

\section{Concept of electrostatics}

Electrostatics is a scientific study and application of electric charges accrued on a surface. The French colonel, Charles Augustin de Coulomb ${ }^{27,28}$ established the fundamental law governing static charges, revealing the behaviour of two point charges in space and their interactions. Electric charge amassed on a sharp pin-tip may be observed as the point charge. Coulomb (C) is a general (SI) unit of electric charge quantification and a charge of one coulomb is nearly comparable to $6.24 \times 10^{18}$ electrons.

Coulomb's law: According to Coulomb's law, the force of pull or repulsion $(F)$ experienced by the point charges $Q_{1}$ and $Q_{2}$ plays along the straight line linking them, and directly proportional to the product of charges and inversely to the square of distance $(R)$ between them. It is expressed as

$$
\begin{aligned}
& F \propto \frac{Q_{1} Q_{2}}{R^{2}}, \\
& F=\frac{K Q_{1} Q_{2}}{R^{2}},
\end{aligned}
$$

where $K=1 / 4 \pi \varepsilon_{0}$ is the proportionality constant, $Q_{1}$ and $Q_{2}$ are point charges (C) in space separated by distance $R(m), F$ the force of attraction or repulsion $(\mathrm{N})$ and $\varepsilon_{0}$ is the permittivity of free space $(\mathrm{F} / \mathrm{m})$.

$$
\varepsilon_{0}=\frac{10^{-9}}{36 \pi}(\mathrm{F} / \mathrm{m})
$$

Therefore

$$
K=\frac{1}{4 \pi \varepsilon_{0}}=9 \times 10^{9} \mathrm{~m} / \mathrm{F} \text { or } \mathrm{N} \mathrm{m}^{2} / \mathrm{C}^{2} .
$$

The particles of homogeneous charge polarity push each other apart. On the contrary, particles with opposite polarity experience a force of attraction between them. The theory of electrostatics is applicable for both conducting as well as non-conducting materials like plastic. Being a part of the plant body, pollen grains must have some water content within them.

Polar nature of water molecules: Water is one of the stable compounds found in nature. However, it has a polar molecular structure with an oxygen atom on one side and two hydrogen atoms on the opposite side, which generates an electric dipole moment at the molecular level $^{29}$. On the basis of electrochemistry of polar compounds, aqueous droplets could be imposed with static charge and therefore conceivably pollen grains also.

Honey-bee charge: Researchers have studied the role of electrostatic forces in natural pollination by honey bees. This has revealed the notion of contactless release of pollen from anther under the influence of electrostatic forces accrued on the body of the honey bee hovering over a flower ${ }^{22}$. Involvement of such a force has been 
confirmed by researchers ${ }^{30-33}$, which can be applied in artificial pollination.

This concept can be applied for both cases, i.e. for noncontact detachment of pollen from the anther as well as deposition of pollen on the targets more effectively. The non-contact detachment method using electrostatic forces may reduce physical damage to the pollen grains and flowers, enabling safe and healthy pollination which ensures higher yield, better quality and expected market price. Further studies are needed regarding the force required for safe non-contact detachment of pollen from the anther, which is a significant factor for designing such equipment. This required force may vary with respect to plant families and species.

Electrostatic charge on plants: At a first glance, one would be certain that the plants being grouted to the earth must be at null electric potential. In nature, however, a slight negative electric pole builds up due to metabolic progression of an active plant body. The observations have shown that the free-falling pollens get attracted or slightly drifted towards receptive stigma, which confirms the electrostatic interaction between pollens and female part of flower ${ }^{22}$.

\section{Design of electrostatic charging device}

The device can be designed for both electrostatic non-contact detachment as well as electro-deposition of pollen by gathering information regarding major parameters.

Electrostatic non-contact pollen detachment deviceconcept: The electrostatic force generated by the device should have a value just a little above the detachment force required for the particular plant species to ensure safe removal and minimum physical damage to the pollen grains and stamen. Calculation of required detachment force is done by the protruding high voltage direct current (HVDC) electrode, which may be able to exert detaching force on the anther by carrying opposite charge in a predetermined electrical field. The electrical field intensity can be increased by increasing voltage potential or by reducing distance between electrode and grounded anther or vice versa. By knowing the detachment force, one can develop electric field near the anther using suitable equipment to exert predetermined electrostatic force which may detach the pollen successfully without actual contact (Figure 2).

If the electrical field intensity $(E)$ and radius of pollen grain $\left(R_{\mathrm{p}}\right)$ are known, then charge induced $\left(Q_{\mathrm{p}}\right)$ on a pollen grain can be calculated as

$$
Q_{\mathrm{p}}=1.65\left(4 \pi \varepsilon_{0} R_{\mathrm{p}}^{2} E\right)
$$

Therefore, the force $\left(F_{\mathrm{p}}\right)$ experienced by the pollen grains can be calculated using the formula

$$
F_{\mathrm{p}}=E \times Q_{\mathrm{p}}
$$

Electrostatic charging of pollen grain and deposition: The device which may charge the pollen grains can be a spray nozzle or a simple orifice made up of suitable material. There are certain methods to charge the particulate matter.

Contact or conduction charging: The contact charging involves the direct conductive contact of the material to be charged and high potential voltage, in which charge transfer is done by conduction (Figure 3 ). It requires a high voltage source, conductive nozzle and media (usually liquid) to transfer the charge. The high voltage terminal is connected to the nozzle through which material flows out and the other terminal is grounded to the earth. As soon as the material starts to flow through the charged nozzle, conductive charge transfer takes place from the nozzle to the material. Due to direct contact between the charged nozzle and material, there is a sudden voltage drop in the charging system which interrupts the charge transfer process and causes uneven charging of particles. Also, there may be a chance of corrosion while using highvoltage conductive metal nozzle in contact with liquid medium $^{34,35}$.

Ionized-field or corona charging: The ionized field or corona could be observed as soon as the electric potential

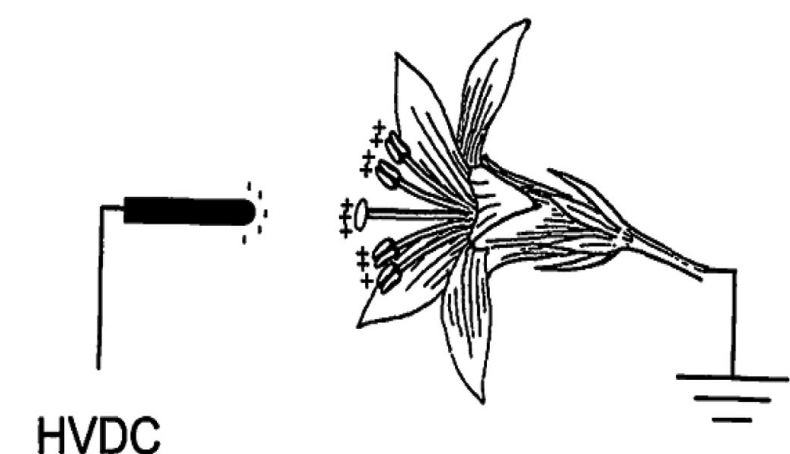

Figure 2. Detachment force measurement (source: ref. 22).

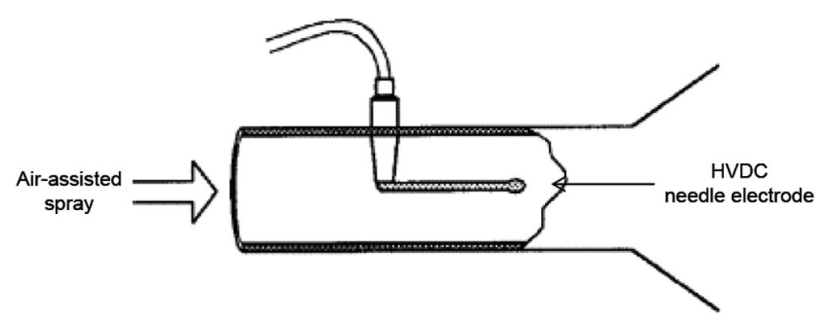

Figure 3. Contact charging method (source: ref. 50). 


\section{REVIEW ARTICLES}

gradient at the probe is high enough to ionize the surrounding air, but not surged adequately to produce electric discharge (Figure 4). The ionized plasma region is chemically active and releases light similar to a discharge $\operatorname{lamp}^{36}$

Since, agricultural spray materials often consist of a solution of chemicals with water as the solvent, corona charging may interfere with their chemical properties and lead to undesirable changes. Therefore, the use of corona charging for agricultural sprays is limited to nonconducting spray materials done. Also, higher voltage used to generate ionized field seems to be hazardous to life.

Triboelectric charging: The Greek word 'tribo-' is synonymous to friction, conveying that the two constituents must be in surface friction to exhibit electron-exchange, and thereby a static charge transformation. The frictive surface contact forms a adhesive bonding between two substances, which allows free movement of electrons from one material to the other, and vice versa. On separation, a few superficially bonded atoms reflect affinity to trap surplus electrons, while a few atoms lose them to the earlier ones. Therefore, electron imbalance within the system makes both the material surfaces electrically charged, one positive while the other negative, and exhibits the well-known Coulombian forces of attraction and repulsion ${ }^{37,38}$.

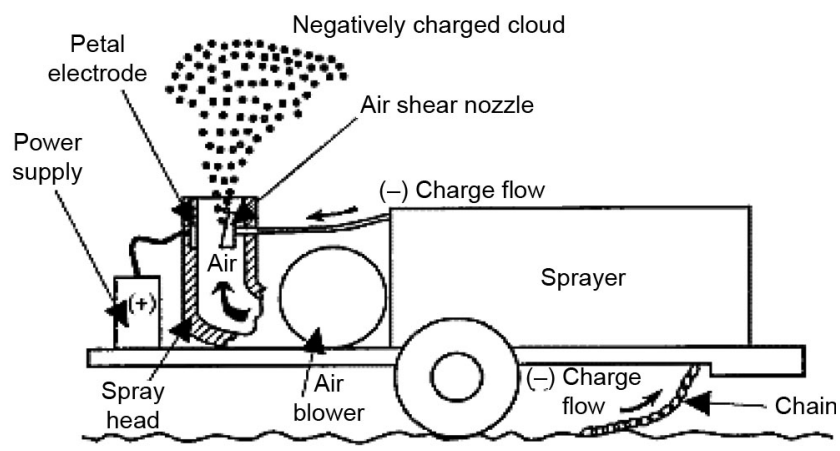

Figure 4. Ionized field charging method (source: ref. 51).

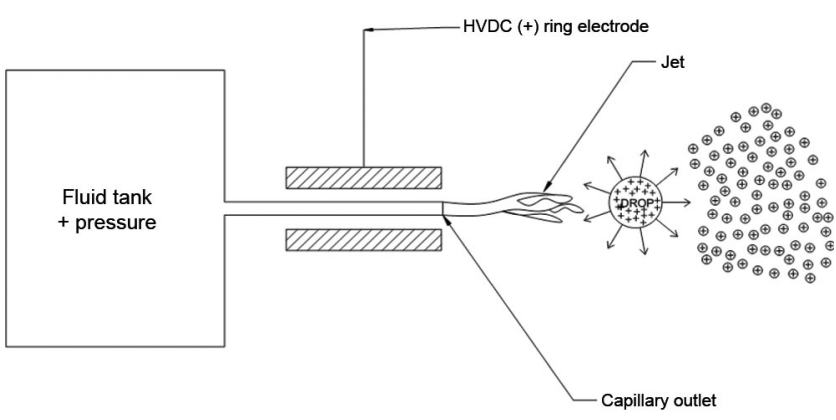

Figure 5. Electro-hydrodynamic spray charging method (source: ref. 40).
Electro-hydrodynamic charging: Electro-hydrodynamic (EHD) is a method of atomizing spray liquids by means of electro-mechanical shear stress along a charging jet surface (Figure 5). This excessive tensions stretches the liquid surface radially emerging into a cone at the tip of the capillary orifice. On the surge of surrounding electrical field strength, this conical liquid sheet deforms into wave perturbations and ultimately disrupts into ligaments to finer droplets. Since the entire process of atomization is carried within and by the high-voltage electric field, eventually the droplets generated through EHD carry static charge ${ }^{39,40}$.

Electrostatic induction charging: The term 'induction' refers to the phenomenon of influencing an object without any physical contact. In terms of electrostatic induction, a charged body which when placed in an electric field near another uncharged or electrically neutral object will induce equal charge with opposite polarity on that object (Figure 6). The requirement of centrally located electrode inside the spray nozzle as in the case of contact charging can be eliminated by induction charging method, as the induction electrode can be located around the spray nozzle where atomization takes place. Since there is no conductive contact between the spray nozzle and HVDC electrode; there is no electric hazard to the operator $^{41-44}$.

Among all the methods discussed for electrostatic charging of particulate matter or finely atomized spray, the electrostatic induction charging method seems to be best suited for conductive agricultural sprays like pesticide application and pollen grains for electrostatic pollination $^{45-47}$. The charging method is of non-contact and chemically non-interfering type. Hence it can be used for both detachment and deposition of pollen grains, as the electrostatically charged particles show higher deposition levels and lesser off-target movement.

Neutralization process and dispersal time: The process by which aerial particles drop their charge imbalance and achieve equilibrium is known as electrostatic neutralization.

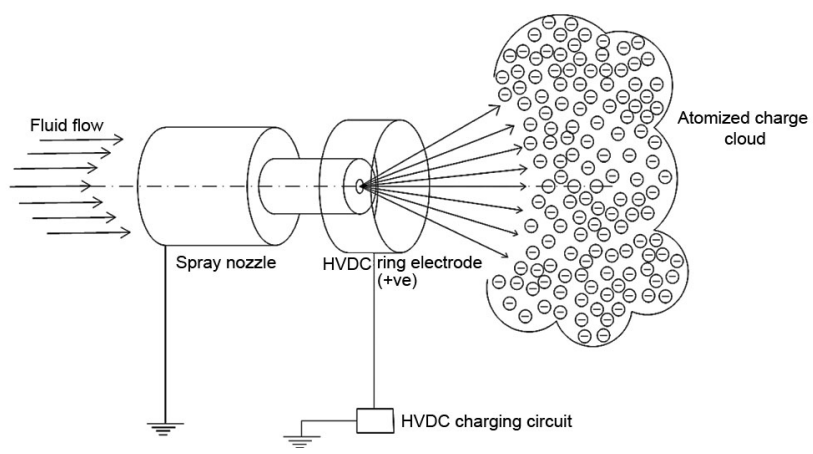

Figure 6. Electrostatic induction charging method (source: ref. 52).

CURRENT SCIENCE, VOL. 120, NO. 3, 10 FEBRUARY 2021 
Since in nature air has relatively lesser electrical conductivity, the process of neutralization consumes a substantial amount of time. In general, for an airborne particle to drop $63 \%$ of its static charge, it takes about $440 \mathrm{~s}$ on an average $^{23,24}$. The dispersal time is the time taken by pollen grains to descend to the ground through air, which is usually much less than that of the neutralization time. This depends on the terminal velocity of the particle and the free-fall height. Hence, if the dispersal time is substantially shorter compared to neutralization time, then it is conceivable that the pollen may possess significant charge through the dispersal period.

Therefore, concept of electrostatically charged pollen deposition can be effectively utilized in artificial pollination with suitable carrying media.

Electrostatic pollination at present: At present, electrostatic pollination method is routinely used for date palms in Arab countries, for larch in forestry and pistachio pollination, etc.

(A) Autonomous date-palm pollinator: This works on blower mechanism with nozzles and charging electrodes connected the HVDC power supply ranging from 20 to $40 \mathrm{kV}$. It is equipped with an autonomous blow direction system which controls the spray and changes the rotating blower head direction with respect to plant position marked by sensors. This system facilitates faster working, and lesser pollination material and less labour requirement too. The pollinator can be installed on tractor as well as utility vehicle.

(B) Electrostatic duster for larch: This pollinator is a tractor-mounted system comprising three major parts. The first component is a front-mounted pollinator chamber intended to cover the entire plant canopy. The second is the electrostatic dust scattering mechanism, and the third is the energizer or high voltage power supply system (Figure 7).

The electrostatic dust scattering mechanism includes three subsystems: (i) electronic control unit (ECU) placed

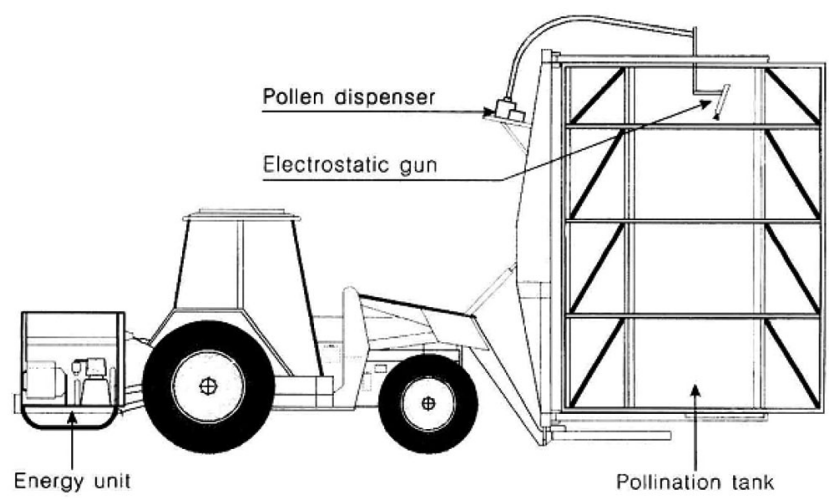

Figure 7. Electrostatic duster for larch (source: ref. 48). inside the tractor-operator cabin, to adjust the energizing levels, pressure and flow rate of the pollen carrier air stream; (ii) pollen conveyer mounted on the pollinator chamber and (iii) electrostatic pollen applicator jet (Ransburg-Gema, model AP 761) clipped on and driven by a gyrating arm. For larch pollination, approximately $1.25 \mathrm{~g}$ of pollen is dispersed in one revolution of applicator arm, using dry air stream at 1 bar as a carrier medium. The energizer unit employ a combined cascade system for the adjustable HVDC output in the range 15-80 kV (refs 48, 49).

(C) Electrostatic pollinating pistol for larch: The selected pollen is applied using a portable electrostatic pistol (Gema Voltstatic model 11024, ITW Gema, Indianapolis, USA) adapted from the model developed by Philippe and Baldet $^{49}$. The pistol and its accompanying equipment are attached to a cart designed by the Forestry, Research Directorate to make easy rapid moves within the tunnel.

\section{Discussion}

This extensive review of the past literature and research outcomes shows the prominent future of commercial applications of electrostatic artificial pollination technology (E-APT). Owing to decline in the population of natural pollinators in the world, this technique seems to be the saviour of the global food security and food-supply chain. In addition, both the crop yield per unit sown area and quality of produce could be enhanced fundamentally by precise application of electrostatic artificial pollination. From the economic point of view, in contrast to conventional hand pollination method, the process of artificial pollination using electrostatic charging would disperse lesser pollen mass to pollinate similar number of plants. Moreover, witnessing the present scarcity of farm workforce scenario and future demands, E-APT could become the foremost tool in future commercial agriculture.

\section{Conclusion}

The following conclusions can be drawn: (i) Electrostatic pollination could reduce the pollen requirement to onethird of conventional artificial pollination practice. (ii) Depositional advantages of charged spraying in agriculture could also be availed though E-APT. (iii) Enhanced pollen deposition over the targeted flower parts could boost fruit set by 1.5 to three-fold. (iv) Electrostatic pollination could double the full seed percentage without reduction in viability. (v) Challenges of future pollinator scarcity could be met to ensure global food security. (vi) It is the need of the hour to bring in expertise from both 
plant breeding and agricultural engineering fraternity to develop a versatile and cost-effective E-APT system.

1. Klein, A. M., Vaissiere, B. E., Cane, J. H., Steffan-Dewenter, I., Cunnigham, S. A., Kremen, C. and Tscharntke, T., Importance of pollinators in changing landscapes for world crops. Proc. R. Soc. London B, 2007, 274, 303-313; doi:10.1098/rspb.2006.3721.

2. Lawrence, T., Pollination and protecting bees and other pollinators. Extension - Washington State University, USA, 2015, pp. 1-9; doi:10.13140/RG.2.1.3099.1203.

3. Ollerton, J., Winfree, R. and Tarrant, S., How many flowering plants are pollinated by animals? Oikos Forum, 2011, 120, 321326; doi:10.1111/j.1600-0706.2010.18644.x.

4. Linder, H. P., Morphology and the evolution of wind pollination. Reprod. Biol. R. Bot. Gard., 1998, 123-135.

5. Friedman, J. and Barrett, H., Wind of change: new insights on the ecology and evolution of pollination and mating in windpollinated plants. Ann. Bot., 2009, 103, 1515-1527.

6. Shapiro, A., Korkidi, E. and Demri, A., Toward elevated agrobotics: development of a scaled-down prototype for visually guided date palm tree sprayer. J. Field Robot., 2009, 26(6-7), $572-590$.

7. Timerman, D. Pollen clumping and release mechanisms in wind pollinated plants. M Sc (Biol.) thesis, Concordia University, Canada, 2013, p. 106.

8. Hilmi, M., Bradbear, N. and Mejia, D., Beekeeping and Sustainable Livelihoods, Food and Agriculture Organization of the United Nations, Rome, Italy, 2011, 2nd edn, p. 71.

9. Potts, S. G., Vulliamy, B., Dafni, A., Ne'eman, G. and Willmer, P., Linking bees and flowers: how do floral communities structure pollinator communities? Ecology, 2003, 84, 2628-2642.

10. Yang, C. and Guo, Y., Floral evolution: beyond traditional viewpoint of pollinator mediated floral design. Chinese Sci. Bull. 2005, 50(21), 2413-2417.

11. Rehel, S., Varghese, A., Bradbear, N., Davidar, P., Roberts, S., Roy, P. and Potts, S. G., Benefits of biotic pollination for nontimber forest products and cultivated plants. Conserv. Soc., 2009, $7,213$.

12. Schowalter, T., Pollination, seed predation and seed dispersal. Insect Ecology-An Ecosystem Approach, Academic Press, 2016, 4th edn, pp. 445-476.

13. Yuan, G., Weihua, M., Wenqing, W. and Huailei, S., Fruit tree pollination technology and industrialization in China. In Beekeeping and Bee Conservation-Advances in Research, 2016, Ch. 6, pp. 153-175, ISBN:978-953-51-2412-2; doi:10.5772/61424.

14. Vaknin, Y., Gan-Mor, S., Bechar, A. and Ronen, B., The role of electrostatic forces in pollination. Plant Syst. Evol., 2000, 222, 133-142.

15. Vaknin, Y., Eisikowitch, D., Gan-Mor, S. and Bechar, A., Electrostatic pollination of pistachio (Pistacia vera L.) - a novel technique of pollen supplementation in agriculture. In 11 th GREMPA Seminar on Pistachios and Almonds. Zaragoza, CIHEAM, Sanliurfa, Turkey, 2001, pp. 53-57; http://om.ciheam.org/article.php?IDPDF= 1600152.

16. Vaknin, Y., Gan-Mor, S., Bechar, A. and Ronen, B., Are flowers morphologically adapted to take advantage of electrostatic forces in pollination? New Phytol., 2001, 151, 301-306.

17. Goodwin, R. M. and Steven, D., Behaviour of honey bees visiting kiwifruit flowers. NZ J. Crop Hortic. Sci., 1993, 21(1), 17-24; doi:10.1080/01140671.1993.9513741.

18. Goodwin, M., Pollination in Australia and New Zealand, Rural Industries Research and Development Corporation, 2012, vol. 12(059), p. 120; ISBN:978-1-74254-402-1.

19. Goodwin, R. M., McBrydie, H. M. and Taylor, M. A., Wind and honey bee pollination of kiwifruit (Actinidia chinensis
'HORT16A'). NZ J. Bot., 51(3), 229-240; doi:10.1080/ 0028825X.2013.806934.

20. Hii, M. J., Kiwifruit flower pollination - wind pollination efficiencies and sprayer jet applications. PhD thesis, University of Canterbury, New Zealand, 2004, p. 323.

21. Luca, P. and Mario, M., The ecology and evolutionary significance of buzz-pollination. Curr. Opin. Plant Biol., 2013, 16, 1-7; http://dx.doi.org/10.1016/j.pbi.2013.05.002.

22. Gan-Mor, S., Schwartzi, Y., Bechar, A. and Eisikowitch, D., Relevance of electrostatic forces in natural and artificial pollination. Can. Agric. Eng., 1995, 37(3), 189-194.

23. Bowker, G. and Crenshaw, H., Electrostatic forces in windpollination - Part 1: measurement of the electrostatic charge on pollen. Atmosp. Environ., 2007a, 41, 1587-1595; http://www. elsevier.com/locate/atmosenv.

24. Bowker, G. and Crenshaw, H., Electrostatic forces in windpollination - Part 2: simulations of pollen capture. Atmos. Environ., 2007b, 41, 1596-1603; http://www.elsevier.com/locate/atmosenv.

25. Gupta, A., Godara1, R., Sharma, R. and Panda, A., Artificial pollination: a tool for improving fruiting traits in date palm (Phoenix dactylifera L.). Chem. Sci. Rev. Lett., 2017, 6(22), 1312-1320.

26. Awad, M. A. Pollination of date palm (Phoenix dactylifera L.) 'Lulu' with pollen grains-water Suspension. Met., Environ Arid Land Agric. Sci., 2011, 22(1) 91-101.

27. Oliveira, A. R., The contribution of coulomb to applied mechanics. In International Symposium on History of Machines and Mechanics, Springer, Dordrecht, The Netherlands, 2004, pp. 217 226; ISBN 978-1-4020-2204-3; https://doi.org/10.1007/1-40202204-2_18

28. Jha, D. K., Text Book of Electrostatics, Discovery Publishing House, New Delhi, 2005, 1st edn, p. 100.

29. Sharp, K., Water: structure and properties. Encyclopedia of Life Sciences, pp. 1-7.

30. Zakon, H., Electric fields of flowers stimulate the sensory hairs of bumble bees. Proc. Natl. Acad. Sci. USA, 2016, 113(26), 7020 7021.

31. Clarke, D., Whitney, H., Sutton, G. and Robert, D., Detection and learning of floral electric fields by bumblebees. Science, 2013, 340(6128), 66-69; doi:10.1126/science.1230883.

32. Badger, M., Victor, O. and Ashley, S. Electrostatic charge on flying hummingbirds and its potential role in pollination. University of Nebraska, 2015, p. 11.

33. Clarke, D., Morley, E. and Robert, D., The bee, the flower, and the electric field: electric ecology and aerial electroreception. $J$. Comp. Physiol., 2017, 203(9), 737-748.

34. Zhao, S., Peter, G. S. and Adamiak, K., Comparison of conduction and induction charging in liquid spraying. J. Electrostat., 2005, 63(6-10), 871-876.

35. Pelesz, A. and Czapka, T., Empirical and numerical analysis of conduction and induction charging of droplets in a three-electrode system. Energies, 2020, 13(469), 1-15; doi:10.3390/en13020469.

36. Yu, R., Zhou, H. and Zheng, J., Design and experiments on droplet charging device for high range electrostatic sprayer. In Pesticides in Modern World - Pesticide Use and Manage., pp. 137-148; doi:10.5772/950

37. Williams, M. W., Triboelectric charging of insulating polymers some new perspectives. AIP Adv., 2012, 2, 5200-5207.

38. Landauer, J. and Forest, P., Influence of particle contact number on triboelectric separation selectivity. Processes, 2019, 7(716), 1-13; doi:10.3390/pr7100716.

39. Luciana, N. and Cramariuc, R., Contribution about the electrohydrodynamic spraying. UPB Sci. Bull., Series C, 2009, 71(3), 205213.

40. Xie, J., Jiang, J., Davoodi, P., Srinivasan, M. and Wang C., Electrohydrodynamic atomization: a two-decade effort to produce and process micro-/nanoparticulate materials. Chem. Eng. Sci., 2015, $125,32-57$. 


\section{REVIEW ARTICLES}

41. Smith, D. B., Goering, C. E., Liljeldahl, L. A. and Reichard, D. L. AC charging of Agricultural sprays. Trans. ASAE, 1977, 34(6), 1002-1007.

42. Anantheswaran, R. C. and Law, S. E., Electrostatic spraying of turf grass. US Golf Assoc., Green Section Project Rec., 1979, 17(6), 1-4.

43. Law, S. E. and Michael, D. L., Electrostatic deposition of pesticide spray onto foliar targets of varying morphology. Trans. ASAE, 1981, 1441-1445.

44. Mamidi, V., Ghanashyam, C., Patel, M. K., Reddy, V. and Kapur, P., Electrostatic hand pressure swirl nozzle for small crop growers. Int. J. Appl. Sci. Techol., Res. Excellence, 2012, 2(2), 164-168.

45. Marchewicz, A., Sobczyk, A. T., Krupa, A. and Jaworek, A., Electrostatic charging of water spray by induction. J. Phys., Conf. Ser., 2019, 1322(012032), 1-5.

46. Khatawkar, D. S., Dhalin, D., James, P. S. and Subhagan, S. R., Electrostatic induction spray-charging system (embedded electrode) for knapsack mist-blower. Curr. J. Appl. Sci. Technol., 2020, 39(5), 80-91; https://doi.org/10.9734/cjast/2020/v39i530549.

47. Khatawkar, D. S., Dhalin, D. and James, P. S., Electrostatic conversion kit for conventional knapsack mist-blower: development and performance evaluation. Int. J. Curr. Microbiol. App. Sci.,
2020, 9(3), 2227-2242; https://doi.org/10.20546/ijcmas.2020. 903.254 .

48. Philippe, G. and Baldet, P., Electrostatic dusting: an efficient technique of pollination in larch. Ann. Sci. Forest., 1997, 54(3), 301-310.

49. Monique, P. and Lemay, P., A novel approach for the operational production of hybrid larch seeds under northern climatic conditions. For. Chronicle, 2008, 84(1) 95-104.

50. Bowen, H. D., Hebblethwaite, P. and Carleton, W., Application of electrostatic charging to the deposition of insecticides and fungicides on plant surfaces. Agric. Eng., 1952, 33(6), 347-350.

51. Castle, G. S. P., Inculet, I. I., Menzies, D. R. and Frank, R., Deposition studies with a novel form of electrostatic crop sprayer. J. Electrostat., 1981, 10, 65-72.

52. Law, S. E., Embedded-electrode electrostatic induction spray charging nozzle: theoretical and engineering design. Trans. ASAE, 1978, 21(10), 1096-1104.

Received 21 December 2018; revised accepted 10 September 2020

doi: $10.18520 / \mathrm{cs} / \mathrm{v} 120 / \mathrm{i} 3 / 484-491$ 